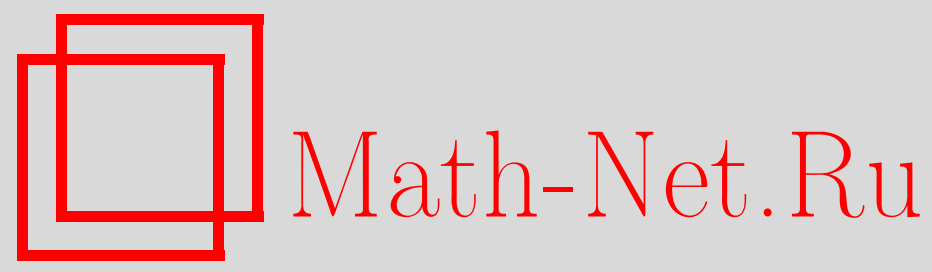

Е. В. Соколовская, О. П. Филатов, Аппроксимация сверху систем дифференциальных включений с нелипшицевой правой частью, Матем. заметки, 2005, том 78, выпуск $5,763-772$

DOI: https://doi.org/10.4213/mzm2632

Использование Общероссийского математического портала Math-Net.Ru подразумевает, что вы прочитали и согласны с пользовательским соглашением http://www.mathnet.ru/rus/agreement

Параметры загрузки:

IP : 34.229 .108 .108

26 апреля 2023 г., 18:30:35






\title{
АППРОКСИМАЦИЯ СВЕРХУ СИСТЕМ ДИФФЕРЕНЦИАЛЬНЫХ ВКЛЮЧЕНИЙ С НЕЛИПШИЦЕВОЙ ПРАВОЙ ЧАСТЬЮ
}

\author{
Е.В. Соколовская, О.П. Филатов
}

Пусть $\mathbb{R}^{n}$ - $p$-мерное пространство с евклидовой нормой $\|\cdot\|, K\left(\mathbb{R}^{p}\right)$ - совокупность непустых компактов в $\mathbb{R}^{p}, \mathbb{R}_{+}=[0,+\infty), D=\mathbb{R}_{+} \times \mathbb{R}^{m} \times \mathbb{R}^{n} \times[0, a], D_{0}=\mathbb{R}_{+} \times \mathbb{R}^{m}$, $F_{0}: D_{0} \rightarrow K\left(\mathbb{R}^{m}\right)$, со $F_{0}$ - выпуклая оболочка отображения $F_{0}$. Рассматривается задача Коши для системы дифференциальных включений

$$
\dot{x} \in \mu F(t, x, y, \mu), \quad \dot{y} \in G(t, x, y, \mu), \quad x(0)=x_{0}, \quad y(0)=y_{0}
$$

с медленными - $x$ и быстрыми - $y$ переменными; здесь $F: D \rightarrow K\left(\mathbb{R}^{m}\right), G: D \rightarrow K\left(\mathbb{R}^{n}\right)$, $\mu \in[0, a]$ - малый параметр. Предполагается, что эта задача имеет хотя бы одно решение на $[0,1 / \mu]$ при всех достаточно малых $\mu \in[0, a]$. При некоторых условиях на $F, G$ и $F_{0}$, как обычных для задач аппроксимации, так и новых (более слабых, чем липшицевость) доказывается, что для любого $\varepsilon>0$ существует $\mu_{0}>0$ такое, что для любого $\mu \in\left(0, \mu_{0}\right]$ и любого решения $\left(x_{\mu}(t), y_{\mu}(t)\right)$ рассматриваемой задачи найдется решение $u_{\mu}(t)$ задачи $\dot{u} \in \mu$ со $F_{0}(t, u), u(0)=x_{0}$, для которого при каждом $t \in[0,1 / \mu]$ вьполняется неравенство $\left\|x_{\mu}(t)-u_{\mu}(t)\right\|<\varepsilon$.

Библиография: 8 названий.

1. Введение и обозначения. Пусть $\mathbb{R}^{n}-n$-мерное евклидово пространство со скалярным произведением $\langle\cdot, \cdot\rangle$ и соответствующей нормой $\|\cdot\|, K\left(\mathbb{R}^{n}\right)$ - совокупность всех непустых компактов из $\mathbb{R}^{n}, K v\left(\mathbb{R}^{n}\right)$ - совокупность всех непустых выпуклых компактов из $\mathbb{R}^{n}, \rho(a, B)$ - расстояние от точки $a \in \mathbb{R}^{n}$ до множества $B \in K\left(\mathbb{R}^{n}\right)$, $\beta(A, B)=\sup _{a \in A} \rho(a, B)-$ полуотклонение по Хаусдорфу множества $A$ от множества $B, \alpha(A, B)=\max \{\beta(A, B), \beta(B, A)\}$ - расстояние по Хаусдорфу между компактами $A$ и $B, \mathbb{R}_{+}=[0,+\infty), L A\left(\mathbb{R}_{+}\right)$- совокупность локально интегрируемых (по Лебегу) функций $\lambda: \mathbb{R}_{+} \rightarrow \mathbb{R}_{+}$, для которых найдутся константы $c_{\lambda}$ и $\Delta_{\lambda}$ такие, что при $\Delta>\Delta_{\lambda}$ для каждого $t_{0} \in \mathbb{R}_{+}$вьполняется неравенство

$$
\frac{1}{\Delta} \int_{t_{0}}^{t_{0}+\Delta} \lambda(t) d t<c_{\lambda} .
$$

Кроме того, пусть $H[0, b], b>0,-$ совокупность всех функций $l:[0, b] \rightarrow \mathbb{R}_{+}$, непрерывных в 0 , для которых $l(0)=0$.

Рассмотрим задачу Коши для системы дифференциальных включений с медленными $-x$ и быстрыми $-y$ переменными

$$
\begin{array}{ll}
\dot{x} \in \mu F(t, x, y, \mu), & x(0)=x_{0}, \\
\dot{y} \in G(t, x, y, \mu), & y(0)=y_{0},
\end{array}
$$


где $F: D \rightarrow K\left(\mathbb{R}^{m}\right), G: D \rightarrow K\left(\mathbb{R}^{n}\right), D=\mathbb{R}_{+} \times \mathbb{R}^{m} \times \mathbb{R}^{n} \times[0, a], \mu \in[0, a], a>0,-$ малый параметр.

При аппроксимации сверху задачи (2) ей сопоставляется так называемая усредненная задача Коши

$$
\dot{u} \in \mu F_{0}(t, u), \quad u(0)=x_{0},
$$

где $F_{0}: D_{0} \rightarrow K\left(\mathbb{R}^{m}\right), D_{0}=\mathbb{R}_{+} \times \mathbb{R}^{m}$, достаточно адекватно отражающая основные свойства медленных переменных системы (2).

ОПРЕДЕЛЕНИЕ [1]. Говорят, что задача (3) аппроксимирует сверху задачу (2), если $\forall \varepsilon>0 \exists \mu_{0}>0$ такое, что для любого $\mu \in\left(0, \mu_{0}\right]$ и любого решения $\left(x_{\mu}(t), y_{\mu}(t)\right)$ задачи $(2)$ найдется решение $u_{\mu}(t)$ задачи (3), для которого выполняется неравенство

$$
\left\|x_{\mu}(t)-u_{\mu}(t)\right\|<\varepsilon \quad \forall t \in\left[0, \frac{1}{\mu}\right] .
$$

Вопрос об аппроксимации сверху задачи (2) изучен в случае, когда правые части дифференциальных включений задачи (2) и отображение $F_{0}$ являются липшицевыми отображениями. В этом случае при условии выпуклости $F_{0}(t, u)$ и некоторых дополнительных предположениях, естественных для задач аппроксимации, доказано [2], [3], что задача (3) апшроксимирует сверху задачу (2). Результаты по аппроксимации сверху в липшицевом случае при отсутствии быстрых переменньх изложены в [4]. Однако стандартная процедура усреднения, позволяющая в ряде случаев найти вид задачи (3), может привести к усредненной задаче с нелипшицевой правой частью. Настоящая работа посвящена этому случаю. Доказывается, что при выполнении для отображений $F$, $G$ и $F_{0}$ некоторых условий, как обычных для задач аппроксимации, так и новых (более слабых, чем липшицевость), задача (2) будет аппроксимироваться сверху задачей

$$
\dot{u} \in \mu \operatorname{co} F_{0}(t, u), \quad u(0)=x_{0},
$$

где со $F_{0}$ - вьпуклая оболочка отображения $F_{0}$. Заметим, что возможность аппроксимации сверху сингулярно возмущенных дифференциальных включений с медленными и быстрыми переменными в не липшицевом случае при других условиях доказана в [5].

2. Основные условия. Будем считать, что имеют место следующие условия.

a) $F(t, x, y, \mu), G(t, x, y, \mu)$ и $F_{0}(t, u)$ измеримы по $t$ на $\mathbb{R}_{+}$соответственно для любых $(x, y, \mu) \in \mathbb{R}^{m} \times \mathbb{R}^{n} \times[0, a]$ и любых $u \in \mathbb{R}^{m}$.

б) Отображения $F, G$ и $F_{0}$ интегрально ограничены функциями $\lambda_{F}, \lambda_{G}, \lambda_{F_{0}} \in$ $L A\left(\mathbb{R}_{+}\right)$, т.е.

$$
\begin{gathered}
\|F(t, x, y, \mu)\| \leqslant \lambda_{F}(t), \quad\|G(t, x, y, \mu)\| \leqslant \lambda_{G}(t) \quad \forall(t, x, y, \mu) \in D ; \\
\left\|F_{0}(t, u)\right\| \leqslant \lambda_{F_{0}}(t) \quad \forall(t, u) \in D_{0} .
\end{gathered}
$$

в) Условие близости средних для $F$ и $F_{0}$ :

$$
\lim _{\Delta \rightarrow \infty} \beta\left(\bigcup \frac{1}{\Delta} \int_{t_{0}}^{t_{0}+\Delta} F\left(t, \xi_{0}, \eta(t), 0\right) d t, \frac{1}{\Delta} \int_{t_{0}}^{t_{0}+\Delta} F_{0}\left(t, \xi_{0}\right) d t\right)=0
$$


равномерно по начальным условиям $t_{0} \in \mathbb{R}_{+}, \xi_{0} \in \mathbb{R}^{m}, \eta_{0} \in \mathbb{R}^{n}$ задачи

$$
\dot{\xi}=0, \quad \dot{\eta} \in \operatorname{co} G(t, \xi, \eta, 0), \quad \xi\left(t_{0}\right)=\xi_{0}, \quad \eta\left(t_{0}\right)=\eta_{0}
$$

а объединение в (5) берется по всем решениям $\eta(t)$ этой задачи.

г) Для любых $r \in[0, b]\left(b>0\right.$-некоторое число), $t \in \mathbb{R}_{+}$и $\left(x_{1}, y_{1}\right),\left(x_{2}, y_{2}\right) \in \mathbb{R}^{m} \times \mathbb{R}^{n}$, $u_{1}, u_{2} \in \mathbb{R}^{m}$ таких, что $\left\|x_{1}-x_{2}\right\| \leqslant r,\left\|y_{1}-y_{2}\right\| \leqslant r,\left\|u_{1}-u_{2}\right\| \leqslant r$ имеют место оценки

$$
\begin{gathered}
\alpha\left(F\left(t, x_{1}, y_{1}, 0\right), F\left(t, x_{2}, y_{2}, 0\right)\right) \leqslant \sigma_{F}(t) \cdot \omega_{F}(r), \\
\alpha\left(G\left(t, x_{1}, y, 0\right), G\left(t, x_{2}, y, 0\right)\right) \leqslant \sigma_{G}(t) \cdot \omega_{G}(r) \quad \forall y \in \mathbb{R}^{n}, \\
\alpha\left(F_{0}\left(t, u_{1}\right), F_{0}\left(t, u_{2}\right)\right) \leqslant \sigma_{F_{0}}(t) \cdot \omega_{F_{0}}(r)
\end{gathered}
$$

с положительными функциями $\sigma_{F}, \sigma_{G}, \sigma_{F_{0}} \in L A\left(\mathbb{R}_{+}\right)$и $\omega_{F}, \omega_{G}, \omega_{F_{0}} \in H[0, b]$.

д) Найдутся функции $\kappa_{F}, \kappa_{G} \in H[0, b]$ такие, что для любых $(t, x, y, \mu) \in \mathbb{R}_{+} \times \mathbb{R}^{m} \times$ $\mathbb{R}^{n} \times[0, b]$ вьполняются неравенства

$\beta(F(t, x, y, \mu), F(t, x, y, 0)) \leqslant \sigma_{F}(t) \cdot \kappa_{F}(\mu), \quad \beta(G(t, x, y, \mu), G(t, x, y, 0)) \leqslant \sigma_{G}(t) \cdot \kappa_{G}(\mu)$.

Следуя [6], будем назьвать многозначное отображение $E: D_{0} \rightarrow K\left(\mathbb{R}^{m}\right)$ односторонне липиицевым (OSL) по $x$, если найдется локально интегрируемая (по Лебегу) в $\mathbb{R}_{+}$функция $l_{E}: \mathbb{R}_{+} \rightarrow \mathbb{R}_{+}$такая, что $\forall x, y \in \mathbb{R}^{m}, \forall t \in \mathbb{R}_{+}, \forall v \in E(t, x)$ существует такой вектор $w \in E(t, y)$, что $\langle x-y, v-w\rangle \leqslant l_{E}(t)\|x-y\|^{2}$.

Для однозначных функций это условие появилось впервые, по-видимому, в [7] в связи с вопросом о единственности решения задачи Коши для системы обыкновенных дифференциальных уравнений.

Заметим, что если отображение $E$ липшицево по $x$ при каждом $t$ из $\mathbb{R}_{+}$с функцией $l(t)$, локально интегрируемой на $\mathbb{R}_{+}$, то это отображение будет удовлетворять и условию OSL.

Для задачи Коши

$$
\dot{x} \in E(t, x), \quad x(0) \in K_{0},
$$

где $E:[0,1] \times \mathbb{R}^{m} \rightarrow K v\left(\mathbb{R}^{m}\right), K_{0} \in K\left(\mathbb{R}^{m}\right)$ с OSL правой частью $E(t, x)$, как показано в [6], имеет место теорема о непрерывной зависимости решения от исходных данных, которая используется при доказательстве основной теоремы настоящей работы.

\section{3. Основная теорема.}

ТЕОРемА. Пусть задача (2) имеет хотя бы одно решение на $[0,1 / \mu]$ при каждом $\mu \in[0, a]$ и выполнены условия а)-д). Предположим, кроме того, что отображсние $G(t, x, y, 0)$ при каждом $x \in \mathbb{R}^{m}$ удовлетворяет условию OSL по у $c$ постоянной функцией $l_{G}=d$, , отображсение $F_{0}(t, u)$ является OSL по и с функиией $l_{F_{0}}(t)$ такой, что при $\Delta \rightarrow+\infty$

$$
\frac{1}{\Delta} \int_{0}^{\Delta} l_{F_{0}}(t) d t=O(1) .
$$

Тогда задача (4) аппроксимирует сверху задачу (2). 
ЗАмЕчАнИЕ 1 . Если наряду с условием г) от отображения $G(t, x, y, 0)$ потребовать выполнение аналогичного условия по $y: \alpha\left(G\left(t, x, y_{1}, 0\right), G\left(t, x, y_{2}, 0\right)\right) \leqslant \sigma_{G}(t) \cdot \omega_{G}(r)$ $\forall x \in \mathbb{R}^{m}$, где $\sigma_{G}(t) \cdot \omega_{G}(r)$ - функция Камке [8, с. 219], то объединение в условии близости средних (5) можно брать по всем решениям порождающей задачи

$$
\dot{\xi}=0, \quad \dot{\eta} \in G(t, \xi, \eta, 0), \quad \xi\left(t_{0}\right)=\xi_{0}, \quad \eta\left(t_{0}\right)=\eta_{0},
$$

которая получается из задачи (2) при $\mu=0$.

ЗАМЕЧАНИЕ 2 . Если функция $\sigma_{F_{0}}(t) \cdot \omega_{F_{0}}(r)$ из условия г) для отображения $F_{0}(t, u)-$ функция Камке, то задача (2) аппроксимируется сверху задачей (3), так как по теореме [8, с. 219] множество решений задачи (3) плотно в множестве решений задачи (4) по норме пространства $C[0,1 / \mu]$.

4. Доказательство. Заметим сначала, что одновременное выполнение для $F$ и $G$ условий г) и д) равносильно вьполнению условий

$$
\begin{aligned}
\forall \varepsilon>0 \quad \exists \delta>0 & \forall x_{1}, x_{2} \in \mathbb{R}^{m}, \quad \forall y_{1}, y_{2} \in \mathbb{R}^{n}, \quad \forall t \in \mathbb{R}_{+}, \quad \forall \mu \in[0, a]: \\
& \left\|x_{1}-x_{2}\right\|<\delta, \quad\left\|y_{1}-y_{2}\right\|<\delta, \quad 0 \leqslant \mu<\delta \\
& \Longrightarrow \beta\left(F\left(t, x_{1}, y_{1}, \mu\right), F\left(t, x_{2}, y_{2}, 0\right)\right)<\varepsilon \sigma_{F}(t),
\end{aligned}
$$

$\forall \varepsilon>0 \quad \exists \delta>0 \quad \forall x_{1}, x_{2} \in \mathbb{R}^{m}, \quad \forall y \in \mathbb{R}^{n}, \quad \forall t \in \mathbb{R}_{+}, \quad \forall \mu \in[0, a]:$

$$
\left|x_{1}-x_{2}\right| \mid<\delta, \quad 0 \leqslant \mu<\delta \Longrightarrow \beta\left(G\left(t, x_{1}, y, \mu\right), G\left(t, x_{2}, y, 0\right)\right)<\varepsilon \sigma_{G}(t) .
$$

Выполнение для $F_{0}$ условия г) равносильно вьполнению условия

$$
\begin{gathered}
\forall \varepsilon>0 \quad \exists \delta>0 \quad \forall u_{1}, u_{2} \in \mathbb{R}^{m}, \quad \forall t \in \mathbb{R}_{+}: \\
\left\|u_{1}-u_{2}\right\|<\delta \Longrightarrow
\end{gathered}
$$

Пусть $c, \Delta_{0}$ - максимальные из констант, найденных из условия принадлежности функций $\lambda_{F}, \lambda_{G}, \lambda_{F_{0}}, \sigma_{F}, \sigma_{G}, \sigma_{F_{0}}$ классу $L A\left(\mathbb{R}_{+}\right)$, и констант $c_{l}\left(F_{0}\right), \Delta_{l}\left(F_{0}\right)$, найденных из условия

$$
\int_{0}^{\Delta} l_{F_{0}}(t) d t \leqslant c_{l}\left(F_{0}\right) \cdot \Delta, \quad \text { если } \Delta \geqslant \Delta_{l}\left(F_{0}\right) .
$$

Теперь проверим, что задача (4) аппроксимирует сверху задачу (2).

Возьмем произвольное $\varepsilon>0$ и для него найдем число $\delta_{F_{0}}(\varepsilon)>0$ из условия (8). По числу $\gamma=\min \left\{\delta_{F_{0}}(\varepsilon) / 2, \varepsilon\right\} /(2 c+1)$ найдем число $\delta_{F}(\gamma)>0$ из условия $(6)$ и $\Delta_{1}(\gamma)>\Delta_{0}$ из условия (5), т.е. такое, что для любого $\Delta>\Delta_{1}(\gamma)$ выполняется неравенство

$$
\begin{gathered}
\beta\left(\bigcup_{\eta(t)} \frac{1}{\Delta} \int_{t_{0}}^{t_{0}+\Delta} F\left(t, \xi_{0}, \eta(t), 0\right) d t, \frac{1}{\Delta} \int_{t_{0}}^{t_{0}+\Delta} F_{0}\left(t, \xi_{0}\right) d t\right)<\gamma \\
\forall\left(t_{0}, \xi_{0}, \eta_{0}\right) \in \mathbb{R}_{+} \times \mathbb{R}^{m} \times \mathbb{R}^{n} .
\end{gathered}
$$

Теперь зафиксируем $\Delta>\Delta_{1}(\gamma)$ и примем $\nu=\delta_{F} / c \Delta \exp (d \Delta)$. Пусть $\delta_{G}(\nu)>0$ найдено по $\nu$ из условия (7). Положим $\delta=\min \left\{\delta_{F_{0}}(\varepsilon) / 2, \delta_{F}(\gamma), \delta_{G}(\nu), \varepsilon\right\}$, а в качестве искомого $\mu_{0}>0$ возьмем такое, чтобы вьполнялись неравенства

$$
\mu_{0} c \Delta<\delta, \quad \mu_{0}<\min \left\{\delta_{F}(\gamma), \delta_{G}(\nu), \frac{1}{\Delta}\right\} .
$$


Пусть теперь $\mu \in\left(0, \mu_{0}\right]$ и $\left(x_{\mu}(t), y_{\mu}(t)\right)$ - произвольное решение задачи $(2), t_{j}=$ $j \cdot \Delta, j=0,1, \ldots, x_{j}=x_{\mu}\left(t_{j}\right), j=0,1, \ldots, k, k=[1 / \mu \Delta],[a]$ - целая часть числа $a$. Поскольку

$$
x_{\mu}(t)=x_{0}+\mu \int_{0}^{t} v(s) d s,
$$

где $v(s)$ - некоторый селектор отображения $F\left(s, x_{\mu}(s), y_{\mu}(s), \mu\right)$, для любого $t \in$ $\left[t_{j}, t_{j+1}\right], j=0,1, \ldots, k-1$, получим

$\left\|x_{\mu}(t)-x_{j}\right\| \leqslant \mu \int_{t_{j}}^{t}\|v(s)\| d s \leqslant \mu \int_{t_{j}}^{t} \lambda_{F}(s) d s \leqslant \mu \int_{t_{j}}^{t_{j+1}} \lambda_{F}(s) d s \leqslant \mu_{0} c \Delta<\delta \leqslant \delta_{F}(\gamma)$.

Так как число $\delta_{F}(\gamma)$ выбиралось по числу $\gamma$ из условия (6), то

$\beta\left(F\left(t, x_{\mu}(t), y_{\mu}(t), \mu\right), F\left(t, x_{j}, y_{\mu}(t), 0\right)\right)<\gamma \sigma_{F}(t) \quad \forall t \in\left[t_{j}, t_{j+1}\right], \quad j=0,1, \ldots, k-1$.

По теореме о выборе измеримого селектора (см., например, [8, с. 206]) на $\left[t_{j}, t_{j+1}\right]$, $j=0,1, \ldots, k-1$, найдется измеримый селектор $v_{j}^{1}(t) \in F\left(t, x_{j}, y_{\mu}(t), 0\right)$ такой, что

$$
\begin{aligned}
\left\|v(t)-v_{j}^{1}(t)\right\| & =\rho\left(v(t), F\left(t, x_{j}, y_{\mu}(t), 0\right)\right) \\
& \leqslant \beta\left(F\left(t, x_{\mu}(t), y_{\mu}(t), \mu\right), F\left(t, x_{j}, y_{\mu}(t), 0\right)\right)<\gamma \sigma_{F}(t) .
\end{aligned}
$$

При каждом $j=0,1, \ldots, k-1$ рассмотрим теперь на $\left[t_{j}, t_{j+1}\right]$ дифференциальное включение

$$
\dot{\eta} \in \operatorname{co} G\left(t, x_{j}, \eta, 0\right), \quad \eta\left(t_{j}\right)=y_{\mu}\left(t_{j}\right) .
$$

Покажем, что найдется решение $\eta_{j}(t)$ этой задачи такое, что $\forall t \in\left[t_{j}, t_{j+1}\right]$ вьполняется неравенство $\left\|\eta_{j}(t)-y_{\mu}(t)\right\| \leqslant \delta_{F}(\gamma)$. Для этого введем на $[0,1]$ вспомогательную задачу

$$
\dot{a} \in G_{0}(s, a), \quad a(0)=y_{\mu}\left(t_{j}\right),
$$

где отображение $G_{0}(s, a)$ определяется равенством

$$
G_{0}(s, a)=\Delta \operatorname{coG}\left((1-s) t_{j}+s t_{j+1}, x_{j}, a, 0\right), \quad s \in[0,1] .
$$

Заметим, что если $a(s)$ - решение на $[0,1]$ задачи $(10)$, то функция $\eta_{j}(t)=a\left(\left(t-t_{j}\right) /\right.$ $\left.\left(t_{j+1}-t_{j}\right)\right)$ - решение на отрезке $\left[t_{j}, t_{j+1}\right]$ задачи $(9)$. Кроме того, заметим, что из выполнения условия OSL для $G(t, x, y, 0)$ следует, что отображение $\operatorname{co} G(t, x, y, 0)$ тоже OSL по $y$ с той же функцией $l_{\text {со } G}(t, x)=l_{G}(t, x) \equiv d$; поэтому отображение $G_{0}(s, a)$ также OSL по $a$ с функцией $l_{G_{0}}(s) \equiv d \cdot \Delta$.

C помощью теоремы [6] о непрерывной зависимости решения от исходных данных для OSL дифференциальных включений установим разрешимость на отрезке $[0,1]$ задачи (10). С этой целью введем на $[0,1]$ функцию $z(s)=y_{\mu}\left((1-s) t_{j}+s t_{j+1}\right)$. Для нее имеем

$$
\begin{aligned}
& \rho\left(\dot{z}(s), G_{0}(s, z(s))\right)=\rho\left(\Delta \dot{y}_{\mu}\left((1-s) t_{j}+s t_{j+1}\right), G_{0}\left(s, y_{\mu}\left((1-s) t_{j}+s t_{j+1}\right)\right)\right) \\
& \quad=\rho\left(\Delta \dot{y}_{\mu}\left((1-s) t_{j}+s t_{j+1}\right), \Delta \operatorname{co} G\left((1-s) t_{j}+s t_{j+1}, x_{j}, y_{\mu}\left((1-s) t_{j}+s t_{j+1}\right), 0\right)\right) \\
& \quad=\Delta \rho\left(\dot{y}_{\mu}(t), \operatorname{co} G\left(t, x_{j}, y_{\mu}(t), 0\right)\right) \leqslant \Delta \beta\left(\operatorname{co} G\left(t, x_{\mu}(t), y_{\mu}(t), \mu\right), \operatorname{co} G\left(t, x_{j}, y_{\mu}(t), 0\right)\right) \\
& \quad \leqslant \Delta \beta\left(G\left(t, x_{\mu}(t), y_{\mu}(t), \mu\right), G\left(t, x_{j}, y_{\mu}(t), 0\right)\right),
\end{aligned}
$$


где $t=(1-s) t_{j}+s t_{j+1} \in\left[t_{j}, t_{j+1}\right]$. Учитьвая найденную вьше оценку $\left\|x_{\mu}(t)-x_{j}\right\|<\delta$ $\forall t \in\left[t_{j}, t_{j+1}\right], j=0,1, \ldots, k-1$, и вспоминая, что $\delta \leqslant \delta_{G}(\nu)$, а число $\delta_{G}(\nu)$ выбиралось по числу $\nu$ из выполнения условия (7), получим

$$
\rho\left(\dot{z}(s), G_{0}(s, z(s))\right) \leqslant \nu \Delta \sigma_{G}\left((1-s) t_{j}+s t_{j+1}\right) \quad \forall s \in[0,1], \quad j=0,1, \ldots, k-1 .
$$

По теореме о непрерывной зависимости $[6]$ на $[0,1]$ найдется решение $a(s)$ задачи $(10)$, для которого имеет место оценка

$$
\|a(s)-z(s)\| \leqslant \frac{\delta_{F}}{c \exp (d \Delta)} \int_{0}^{s} \exp \left(m_{0}(s)-m_{0}(\tau)\right) \sigma_{G}\left((1-\tau) t_{j}+\tau t_{j+1}\right) d \tau,
$$

где $s \in[0,1]$. Поскольку

$$
m_{0}(s)=\int_{0}^{s} l_{G_{0}}(\alpha) d \alpha \leqslant \int_{0}^{1} l_{G_{0}}(\alpha) d \alpha=\int_{0}^{1} d \Delta d \alpha=\Delta d
$$

TO

$$
\begin{aligned}
\|a(s)-z(s)\| & \leqslant \frac{\delta_{F}}{c \exp (d \Delta)} \int_{0}^{s} \exp \left(m_{0}(s)\right) \sigma_{G}\left((1-\tau) t_{j}+\tau t_{j+1}\right) d \tau \\
& \leqslant \frac{\delta_{F}}{c \exp (d \Delta)} \int_{0}^{1} \exp (d \Delta) \sigma_{G}\left((1-\tau) t_{j}+\tau t_{j+1}\right) d \tau \\
& =\frac{\delta_{F}}{c \Delta} \int_{t_{j}}^{t_{j+1}} \sigma_{G}(\beta) d \beta<\delta_{F}(\gamma) \quad \forall s \in[0,1] .
\end{aligned}
$$

Итак, на $[0,1]$ найдено решение $a(s)$ задачи $(10)$, для которого $\|a(s)-z(s)\|<\delta_{F}(\gamma)$ $\forall s \in[0,1]$. Отсюда следует, что

$$
\left\|a\left(\frac{t-t_{j}}{t_{j+1}-t_{j}}\right)-z\left(\frac{t-t_{j}}{t_{j+1}-t_{j}}\right)\right\|<\delta_{F}(\gamma) \quad \forall t \in\left[t_{j}, t_{j+1}\right], \quad j=0,1, \ldots, k-1 .
$$

Как отмечалось вьше, функция $\eta_{j}(t)=a\left(\left(t-t_{j}\right) /\left(t_{j+1}-t_{j}\right)\right)$ - решение на отрезке $\left[t_{j}, t_{j+1}\right]$ задачи $(9)$, а функция $z\left(\left(t-t_{j}\right) /\left(t_{j+1}-t_{j}\right)\right)=y_{\mu}(t)$. Таким образом, на отрезке $\left[t_{j}, t_{j+1}\right]$ найдено решение $\eta_{j}(t)$ задачи $(9)$, для которого

$$
\left\|\eta_{j}(t)-y_{\mu}(t)\right\|<\delta_{F}(\gamma) \quad \forall t \in\left[t_{j}, t_{j+1}\right], \quad j=0,1, \ldots, k-1 .
$$

Так как число $\delta_{F}(\gamma)$ выбиралось по числу $\gamma$ из условия $(6)$, то

$$
\beta\left(F\left(t, x_{j}, y_{\mu}(t), 0\right), F\left(t, x_{j}, \eta_{j}(t), 0\right)\right)<\gamma \sigma_{F}(t) \quad \forall t \in\left[t_{j}, t_{j+1}\right], \quad j=0,1, \ldots, k-1 .
$$

По теореме о выборе измеримого селектора на $\left[t_{j}, t_{j+1}\right]$ найдется селектор $v_{j}^{2}(t) \in$ $F\left(t, x_{j}, \eta_{j}(t), 0\right)$ такой, что

$$
\begin{aligned}
\left\|v_{j}^{1}(t)-v_{j}^{2}(t)\right\| & =\rho\left(v_{j}^{1}(t), F\left(t, x_{j}, \eta_{j}(t), 0\right)\right) \\
& \leqslant \beta\left(F\left(t, x_{j}, y_{\mu}(t), 0\right), F\left(t, x_{j}, \eta_{j}(t), 0\right)\right)<\gamma \sigma_{F}(t) \\
\forall t & \in\left[t_{j}, t_{j+1}\right], \quad j=0,1, \ldots, k-1 .
\end{aligned}
$$


Так как

$$
\frac{1}{\Delta} \int_{t_{j}}^{t_{j+1}} v_{j}^{2}(t) d t \in \frac{1}{\Delta} \int_{t_{j}}^{t_{j+1}} F\left(t, x_{j}, \eta_{j}(t), 0\right) d t \subset \bigcup_{\eta} \frac{1}{\Delta} \int_{t_{j}}^{t_{j+1}} F\left(s, x_{j}, \eta(t), 0\right) d t
$$

где объединение берется по всем решениям $\eta(t)$ задачи (9), опять по упомянутой теореме о выборе измеримого селектора на $\left[t_{j}, t_{j+1}\right]$ найдется измеримьй селектор $v_{j}^{3}(t) \in$ $F_{0}\left(t, x_{j}\right)$ такой, что

$$
\begin{gathered}
\left\|\frac{1}{\Delta} \int_{t_{j}}^{t_{j+1}} v_{j}^{2}(t) d t-\frac{1}{\Delta} \int_{t_{j}}^{t_{j+1}} v_{j}^{3}(t) d t\right\|=\rho\left(\frac{1}{\Delta} \int_{t_{j}}^{t_{j+1}} v_{j}^{2}(t) d t, \frac{1}{\Delta} \int_{t_{j}}^{t_{j+1}} F_{0}\left(t, x_{j}\right) d t\right) \\
\leqslant \beta\left(\frac{1}{\Delta} \int_{t_{j}}^{t_{j+1}} F\left(t, x_{j}, \eta_{j}(t), 0\right) d t, \frac{1}{\Delta} \int_{t_{j}}^{t_{j+1}} F_{0}\left(t, x_{j}\right) d t\right) \\
\leqslant \beta\left(\bigcup_{\eta(t)} \frac{1}{\Delta} \int_{t_{j}}^{t_{j+1}} F\left(t, x_{j}, \eta(t), 0\right) d t, \frac{1}{\Delta} \int_{t_{j}}^{t_{j+1}} F_{0}\left(t, x_{j}\right) d t\right)<\gamma, \\
j=0,1, \ldots, k-1 .
\end{gathered}
$$

Теперь на $[0,1 / \mu]$ введем функцию

$$
v^{3}(t)= \begin{cases}v_{j}^{3}(t), & \text { если } t \in\left[t_{j}, t_{j+1}\right), \quad j=0,1, \ldots, k-1, \\ 0, & \text { если } t \in\left[t_{k}, 1 / \mu\right] .\end{cases}
$$

И пусть $\omega_{\mu}(t)=x_{0}+\mu \int_{0}^{t} v^{3}(s) d s$. Оценим, на сколько $x_{\mu}(t)$ отличается от $\omega_{\mu}(t)$ в узлах $t_{1}, \ldots, t_{k}$. Имеем

$$
\begin{aligned}
& \left\|x_{\mu}\left(t_{j}\right)-\omega_{\mu}\left(t_{j}\right)\right\|=\left\|x_{0}+\mu \int_{0}^{t_{j}} v(s) d s-x_{0}-\mu \int_{0}^{t_{j}} v^{3}(s) d s\right\| \\
& \leqslant\left\|\mu \sum_{i=0}^{j-1} \int_{t_{i}}^{t_{i+1}} v(t) d t-\mu \sum_{i=0}^{j-1} \int_{t_{i}}^{t_{i+1}} v_{i}^{1}(t) d t\right\| \\
& +\left\|\mu \sum_{i=0}^{j-1} \int_{t_{i}}^{t_{i+1}} v_{i}^{1}(t) d t-\mu \sum_{i=0}^{j-1} \int_{t_{i}}^{t_{i+1}} v_{i}^{2}(t) d t\right\| \\
& +\left\|\mu \sum_{i=0}^{j-1} \int_{t_{i}}^{t_{i+1}} v_{i}^{2}(t) d t-\mu \sum_{i=0}^{j-1} \int_{t_{i}}^{t_{i+1}} v_{i}^{3}(t) d t\right\| \\
& \leqslant \mu \sum_{i=0}^{j-1} \int_{t_{i}}^{t_{i+1}}\left\|v(t)-v_{i}^{1}(t)\right\| d t+\mu \sum_{i=0}^{j-1} \int_{t_{i}}^{t_{i+1}}\left\|v_{i}^{1}(t)-v_{i}^{2}(t)\right\| d t \\
& +\mu \sum_{i=0}^{j-1}\left\|\int_{t_{i}}^{t_{i+1}}\left(v_{i}^{2}(t)-v_{i}^{3}(t)\right) d t\right\| \\
& \leqslant \mu \gamma \sum_{i=0}^{j-1} \int_{t_{i}}^{t_{i+1}} \sigma_{F}(t) d t+\mu \gamma \sum_{i=0}^{j-1} \int_{t_{i}}^{t_{i+1}} \sigma_{F}(t) d t+\mu \gamma \Delta j \\
& \leqslant 2 \mu \gamma c \Delta j+\mu \gamma \Delta j \leqslant 2 \mu \gamma \frac{c}{\mu}+\mu \frac{\gamma}{\mu}=\gamma(2 c+1) \leqslant \varepsilon, \quad j=0,1, \ldots, k .
\end{aligned}
$$


Отметим отдельно неравенство

$$
\left\|x_{\mu}\left(t_{j}\right)-\omega_{\mu}\left(t_{j}\right)\right\| \leqslant \gamma(2 c+1), \quad j=0,1, \ldots, k .
$$

Покажем теперь, что на $[0,1 / \mu]$ найдется решение $u_{\mu}(t)$ задачи $(4)$, близкое к функции $\omega_{\mu}(t)$. Для этого введем на $[0,1]$ вспомогательную задачу

$$
\dot{b} \in F_{0}^{1}(s, b, \mu), \quad b(0)=x_{0}, \quad s \in[0,1],
$$

где отображение $F_{0}^{1}:[0,1] \times \mathbb{R}^{m} \times\left(0, \mu_{0}\right] \rightarrow K v\left(\mathbb{R}^{m}\right)$ задается равенством $F_{0}^{1}(s, b, \mu)=$ co $F_{0}(s / \mu, b)$. Заметим, что если функция $b(s)$ - решение на $[0,1]$ задачи $(12)$, то функция $u_{\mu}(t)=b(\mu t)-$ решение на $[0,1 / \mu]$ задачи (4). К задаче (12) применим теорему [6]. C этой целью оценим сначала расстояние $\rho\left(\dot{z}^{*}(s), F_{0}^{1}\left(s, z^{*}(s), \mu\right)\right)$ для почти всех $s \in[0,1]$, взяв

$$
z^{*}(s)=\omega_{\mu}\left(\frac{s}{\mu}\right)=x_{0}+\mu \int_{0}^{s / \mu} v^{3}(\alpha) d \alpha=x_{0}+\int_{0}^{s} v^{3}\left(\frac{\beta}{\mu}\right) d \beta, \quad s \in[0,1] .
$$

Рассмотрим два случая: $s \in\left[\mu t_{j}, \mu t_{j+1}\right), j=0,1, \ldots, k-1$, и $s \in\left[\mu t_{k}, 1\right]$. В первом случае

$$
\begin{aligned}
\rho\left(\dot{z}^{*}(s), F_{0}^{1}\left(s, z^{*}(s), \mu\right)\right) & =\rho\left(v^{3}\left(\frac{s}{\mu}\right), \operatorname{co} F_{0}\left(\frac{s}{\mu}, \omega_{\mu}\left(\frac{s}{\mu}\right)\right)\right)=\rho\left(v_{j}^{3}(t), \operatorname{co} F_{0}\left(t, \omega_{\mu}(t)\right)\right) \\
& \leqslant \rho\left(v_{j}^{3}(t), F_{0}\left(t, \omega_{\mu}(t)\right)\right) \leqslant \beta\left(F_{0}\left(t, x_{j}\right), F_{0}\left(t, \omega_{\mu}(t)\right)\right) \\
& \leqslant \alpha\left(F_{0}\left(t, x_{j}\right), F_{0}\left(t, \omega_{\mu}(t)\right)\right), \quad \text { где } t=\frac{s}{\mu} \in\left[t_{j}, t_{j+1}\right) .
\end{aligned}
$$

Для того, чтобы теперь использовать условие $(8)$ для $F_{0}$, оценим сначала $\left\|x_{j}-\omega_{\mu}(t)\right\|$ на $\left[t_{j}, t_{j+1}\right)$. Учитывая $(11)$, имеем

$$
\begin{aligned}
\left\|x_{j}-\omega_{\mu}(t)\right\| & \leqslant\left\|x_{j}-\omega_{\mu}\left(t_{j}\right)\right\|+\left\|\omega_{\mu}\left(t_{j}\right)-\omega_{\mu}(t)\right\| \leqslant \gamma(2 c+1)+\mu \int_{t_{j}}^{t} \lambda_{F_{0}}(s) d s \\
& \leqslant \gamma(2 c+1)+\mu \int_{t_{j}}^{t_{j+1}} \lambda_{F_{0}}(s) d s \leqslant \gamma(2 c+1)+\mu c \Delta<\frac{\delta_{F_{0}}(\varepsilon)}{2}+\delta \leqslant \delta_{F_{0}}(\varepsilon) .
\end{aligned}
$$

Отсюда

$$
\alpha\left(F_{0}\left(t, x_{j}\right), F_{0}\left(t, \omega_{\mu}(t)\right)\right)<\varepsilon \sigma_{F_{0}}(t) \quad \forall t \in\left[t_{j}, t_{j+1}\right) .
$$

Из (13) теперь следует, что

$$
\rho\left(\dot{z}^{*}(s), F_{0}^{1}\left(s, z^{*}(s), \mu\right)\right)<\varepsilon \sigma_{F_{0}}\left(\frac{s}{\mu}\right) \quad \forall s \in\left[0, \mu t_{k}\right) .
$$

Во втором случае, когда $s \in\left[\mu t_{k}, 1\right]$,

$$
\begin{aligned}
\rho\left(\dot{z}^{*}(s), F_{0}^{1}\left(s, z^{*}(s), \mu\right)\right) & =\rho\left(v^{3}\left(\frac{s}{\mu}\right), \operatorname{co} F_{0}\left(\frac{s}{\mu}, \omega_{\mu}\left(\frac{s}{\mu}\right)\right)\right) \\
& \leqslant \rho\left(v^{3}\left(\frac{s}{\mu}\right), F_{0}\left(\frac{s}{\mu}, \omega_{\mu}\left(\frac{s}{\mu}\right)\right)\right) \\
& =\rho\left(0, F_{0}\left(\frac{s}{\mu}, \omega_{\mu}\left(\frac{s}{\mu}\right)\right)\right)=\left\|F_{0}\left(\frac{s}{\mu}, \omega_{\mu}\left(\frac{s}{\mu}\right)\right)\right\| \leqslant \lambda_{F_{0}}\left(\frac{s}{\mu}\right) .
\end{aligned}
$$


Если теперь ввести на $[0,1]$ функцию

$$
g(s)=\left\{\begin{array}{lll}
\varepsilon \sigma_{F_{0}}(s / \mu), & \text { если } & s \in\left[0, \mu t_{k}\right), \\
\lambda_{F_{0}}(s / \mu), & \text { если } & s \in\left[\mu t_{k}, 1\right]
\end{array}\right.
$$

то $\forall s \in[0,1]$ будет вьполняться неравенство

$$
\rho\left(\dot{z}^{*}(s), F_{0}^{1}\left(s, z^{*}(s), \mu\right)\right) \leqslant g(s) .
$$

Заметим также, что эта функция $g(s)$ интегрируема на $[0,1]$. Поскольку отображение $F_{0}(t, u)$ - OSL по $u$ с функцией $l_{F_{0}}(t)$, отображение со $F_{0}(t, u)$ также удовлетворяет условию OSL по $u$ с той же функцией $l_{F_{0}}(t)$. Поэтому отображение $F_{0}^{1}(s, b, \mu)$ является OSL по $b$ с функцией $l_{1}(s)=l_{F_{0}}(s / \mu)$. В таком случае по теореме [6] найдется на $[0,1]$ решение $b(s)$ задачи $(12)$, для которого при всех $s \in[0,1]$ выполняется неравенство

$$
\left\|b(s)-z^{*}(s)\right\| \leqslant \int_{0}^{s} \exp \left[m_{1}(s)-m_{1}(\tau)\right] g(\tau) d \tau \leqslant \int_{0}^{1} \exp \left[m_{1}(s)\right] g(\tau) d \tau .
$$

Учитывая, что

$$
m_{1}(s)=\int_{0}^{s} l_{1}(\tau) d \tau=\int_{0}^{s} l_{F_{0}}\left(\frac{\tau}{\mu}\right) d \tau=\mu \int_{0}^{s / \mu} l_{F_{0}}(\alpha) d \alpha \leqslant \mu \int_{0}^{1 / \mu} l_{F_{0}}(\alpha) d \alpha \leqslant c,
$$

из (14) находим

$$
\begin{aligned}
\left\|b(s)-z^{*}(s)\right\| & \leqslant \int_{0}^{1} \exp (c) g(\tau) d \tau=\exp (c)\left(\int_{0}^{\mu t_{k}} \varepsilon \sigma_{F_{0}}\left(\frac{\tau}{\mu}\right) d \tau+\int_{\mu t_{k}}^{1} \lambda_{F_{0}}\left(\frac{\tau}{\mu}\right) d \tau\right) \\
& =\exp (c)\left(\varepsilon \mu \int_{0}^{t_{k}} \sigma_{F_{0}}(\alpha) d \alpha+\mu \int_{t_{k}}^{1 / \mu} \lambda_{F_{0}}(\alpha) d \alpha\right) \\
& \leqslant \exp (c)\left(\varepsilon \mu \int_{0}^{1 / \mu} \sigma_{F_{0}}(\alpha) d \alpha+\mu \int_{t_{k}}^{t_{k}+\Delta} \lambda_{F_{0}}(\alpha) d \alpha\right) \\
& \leqslant \exp (c)\left(\frac{\varepsilon \mu c}{\mu}+\mu c \Delta\right) \leqslant \exp (c)(\varepsilon c+\delta) \leqslant \varepsilon(c+1) \exp (c) \quad \forall s \in[0,1] .
\end{aligned}
$$

Следовательно, $\forall t \in[0,1 / \mu]$ выполняется неравенство $\left\|b(\mu t)-z^{*}(\mu t)\right\| \leqslant \varepsilon(c+1) \exp (c)$. Как отмечалось вьше, $b(\mu t)=u_{\mu}(t)$ - решение на $[0,1 / \mu]$ задачи $(4)$, а $z^{*}(\mu t)=\omega_{\mu}(t)$. Поэтому

$$
\left\|u_{\mu}(t)-\omega_{\mu}(t)\right\| \leqslant \varepsilon(c+1) \exp (c) \quad \forall t \in\left[0, \frac{1}{\mu}\right] .
$$

Таким образом, на $[0,1 / \mu]$ найдено решение $u_{\mu}(t)$ задачи $(4)$, для которого вьполняется (15).

Оценим, на сколько найденное решение $u_{\mu}(t)$ отличается от $x_{\mu}(t)$. Возьмем произвольное $t \in(0,1 / \mu]$ и $t_{j}-$ ближайший к точке $t$ слева узел сетки $t_{0}, t_{1}, \ldots, t_{k}$. Имеем

$$
\begin{aligned}
\left\|x_{\mu}(t)-u_{\mu}(t)\right\| \leqslant & \left\|x_{\mu}(t)-x_{\mu}\left(t_{j}\right)\right\|+\left\|x_{\mu}\left(t_{j}\right)-\omega_{\mu}\left(t_{j}\right)\right\| \\
& +\left\|\omega_{\mu}\left(t_{j}\right)-u_{\mu}\left(t_{j}\right)\right\|+\left\|u_{\mu}\left(t_{j}\right)-u_{\mu}(t)\right\|
\end{aligned}
$$




$$
\begin{aligned}
& \leqslant \mu \int_{t_{j}}^{t}\|v(s)\| d s+\varepsilon+\varepsilon(c+1) \exp (c)+\int_{t_{j}}^{t}\left\|\dot{u}_{\mu}(s)\right\| d s \\
& \leqslant \mu \int_{t_{j}}^{t_{j+1}} \lambda_{F}(s) d s+\varepsilon(1+(c+1) \exp (c))+\mu \int_{t_{j}}^{t_{j+1}} \lambda_{F_{0}}(s) d s \\
& \leqslant 2 \mu c \Delta+\varepsilon(1+(c+1) \exp (c)) \\
& \leqslant 2 \delta+\varepsilon(1+(c+1) \exp (c)) \leqslant \varepsilon(3+(c+1) \exp (c)) \quad \forall t \in\left[0, \frac{1}{\mu}\right] .
\end{aligned}
$$

В силу произвольности $\varepsilon$ это означает, что задача (4) аппроксимирует сверху задачу (2). Теорема доказана.

\section{СПИСОК ЦИТИРОВАННОЙ ЛИТЕРАТУРЫ}

[1] Филатов О.П., Хапаев М. М. Усреднение систем дифференциальных включений. М.: Изд-во МГУ, 1998.

[2] Филатов О. П., Хапаев М. М. Усреднение дифференциальных включений с "быстрыми" и "медленными" переменными // Матем. заметки. 1990. Т. 47. №6. С. 102-109.

[3] Филатов О.П., Хапаев М. М. О взаимной $\varepsilon$-аппроксимации решений системы дифференциальных включений и усредненного включения // Матем. заметки. 1990. Т. 47. №5. C. $127-134$.

[4] Плотников В. А., Плотников А. В., Витюк А. Н. Дифференциальные уравнения с многозначной правой частью. Асимптотические методы. Одесса: Астропринт, 1999.

[5] Donchev T., Slavov I. Averaging method for one-sided Lipschitz differential inclusions with generalized solutions // SIAM J. Control Optimization. 1999. V. 37. № 5. P. 1600-1613.

[6] Donchev T., Farkhi E. Stability and Euler approximation of one-sided Lipschitz differential inclusions // SIAM J. Control Optimization. 1998. V. 36. № 2. P. 780-796.

[7] Красносельский М. А., Крейн С. Г. Нелокальные теоремы существования и теоремы единственности для систем обыкновенных дифференциальных уравнений // Докл. АН СССР. 1955. Т. 102. № 1. С. 13-16.

[8] Благодатских В.И., Филиппов А. Ф. Дифференциальные включения и оптимальное управление // Тр. МИАН. 1985. Т. 169. С. 194-252.

Самарский государственный университет 